Disclosure of Interests: None declared

DOI: 10.1136/annrheumdis-2019-eular.7215

\section{THU0408 ACHIEVING DISEASE REMISSION IN AXIAL SPONDYLOARTHRITIS: A TWO-CENTRE RETROSPECTIVE ANALYSIS OF RELEVANT BASELINE PATIENT CHARACTERISTICS}

Thomas Williams $^{1}$, Karl Gaffney ${ }^{2}$, Alison Wadeley ${ }^{3}$, Charlotte Cavill ${ }^{1}$, Mandy Freeth ${ }^{1}$, Alan Brooksby ${ }^{2}$, Raj Sengupta ${ }^{1} .{ }^{1}$ Royal National Hospital for Rheumatic Diseases, Rheumatology, Bath, United Kingdom; ${ }^{2}$ Norfolk and Norwich University Hospital, Norwich, United Kingdom; ${ }^{3}$ Bath Spa University, College of Liberal Arts, Bath, United Kingdom

Background: ASAS-EULAR Axial Spondyloarthritis (AxSpA) guidelines rec ommend that $\mathrm{AxSpA}$ treatment should be guided according to a predefined target ${ }^{1}$, following increasing evidence that persistent, uncontrolled inflammatory disease activity results in long-term damage and poor outcomes $^{2,3}$. Despite this, there remains no single definition for "remission" in $\mathrm{AxSpA}$.

Objectives: To identify baseline characteristics of individuals achieving AxSpA disease "remission" 6 months after initiation of biologic diseasemodifying therapy (bDMARD).

Methods: A two-centre retrospective cross-sectional analysis was performed, of AxSpA patients receiving their first bDMARD at the Royal National Hospital for Rheumatic Diseases, Bath, and Norfolk and Norwich Univeristy Hospital, Norwich. AxSpA "remission" was defined as Bath Ankylosing Spondylitis Disease Activity Index (BASDAI) $<1$; Bath Ankylosing Spondylitis Functional Index (BASFI) $<3.8$ or serum C-reactive protein $(\mathrm{CRP})<5$. A non-parametric (2-tailed Chi-Square) test was applied to identify differences in baseline characteristics which distinguished those patients achieving "remission" after 6 months bDMARD treatment from others.

Results: 538 patients were included. Sufficient BASDAl data was available for 440, CRP data for 448 and BASFI data for 354. Overall, 42 (9.5\%) patients achieved BASDAI $<1 ; 275$ (61.4\%) achieved CRP $<5$ and $206(58.2 \%)$ achieved BASFI <3.8. The differences in baseline characteristics of individuals achieving these AxSpA "remission" outcomes compared with others are summarised in Table 1, with statistically significant results at the $95 \%$ level highlighted.

Abstract THU0408 - Table 1. p-values of 2-tailed non-parametric test comparing baseline characteristics of individuals achieving AxSpA "remission" outcomes after 6 months versus others.

\begin{tabular}{|c|c|c|c|}
\hline \multirow[t]{2}{*}{ Baseline characteristics } & \multicolumn{3}{|c|}{ p-values } \\
\hline & $\begin{array}{c}\text { BASDAI } \\
<1 \\
\mathrm{~N}=42 / 440\end{array}$ & $\begin{array}{c}\text { CRP <5 } \\
N=275 / \\
448\end{array}$ & $\begin{array}{c}\text { BASFI } \\
<3.8 \\
\mathrm{~N}=206 / 354\end{array}$ \\
\hline Lower mean age at axSpA diagnosis & 0.04 & 0.78 & 0.09 \\
\hline $\begin{array}{l}\text { Less mean time from diagnosis to bDMARD } \\
\text { initiation }\end{array}$ & 0.56 & 0.00 & 0.66 \\
\hline Lower median baseline BASDAI & 0.35 & 0.39 & 0.00 \\
\hline Lower median baseline CRP & 0.01 & 0.00 & 0.67 \\
\hline Lower median baseline BASFI & 0.08 & 0.04 & 0.00 \\
\hline Lower median baseline BASMI & 0.73 & 0.44 & 0.00 \\
\hline HLA B27+ & 0.21 & 0.06 & 0.10 \\
\hline Male sex & 0.87 & 0.52 & 0.62 \\
\hline
\end{tabular}

Conclusion: Our self-defined measures of BASDAI and CRP remission were most achievable in our cohort for patients with lower CRP and less functional limitation before bDMARD initiation. This may reflect remission being more achievable when treating milder disease. Functional "remission" was influenced by baseline BASMI and BASDAI in our cohort, highlighting the importance of both disease activity and pre-existing structural damage on functional outcomes. Further work could include longitudinal follow-up of outcomes in these patients to establish the relevance of targeting AxSpA remission. "BASDAI remission" was very narrowly defined in this study, and this analysis could be repeated with a more achievable target eg BASDAI <3.

\section{REFERENCES:}

[1] Van der Heijde D, et al. 2016 update of the ASAS-EULAR management recommendations for axial spondyloarthritis. Ann Rheum Ds 2017; 76 (6): 978-91.

[2] Ramiro S, van der Heijde D, van Tubergen A, et al. Higher disease activity leads to more structural damage in the spine in ankylosing spondylitis: 12- year longitudinal data from the OASIS cohort. Ann Rheum Dis 2014;73:1455-61.

[3] Poddubnyy D, Protopopov M, Haibel H, et al. High disease activity according tothe Ankylosing Spondylitis Disease Activity Score is associated with accelerated radiographic spinal progression in patients with early axia spondyloarthritis: results from the GErman SPondyloarthritis Inception Cohort. Ann Rheum Dis 2016;75:2114-18.

Disclosure of Interests: Thomas Williams: None declared, Karl Gaffney Grant/research support from: Abbvie, Pfizer, Consultant for: Abbvie, Lilly, Novartis, UCB, Speakers bureau: Abbvie, Biogen, Gilead, Lilly, Novartis UCB, Alison Wadeley: None declared, Charlotte Cavill: None declared, Mandy Freeth: None declared, Alan Brooksby: None declared, Raj Sen gupta Grant/research support from: AbbVie, Celgene Corporation, Merck Sharp \& Dohme, Novartis, Pfizer, and UCB, Speakers bureau: AbbVie Celgene Corporation, Merck Sharp \& Dohme, Novartis, Pfizer, and UCB DOI: 10.1136/annrheumdis-2019-eular.4563

\section{THU0409 PATTERN AND INFLUENTIAL FACTORS IN PROMOTING TREAT-TO-TARGET (T2T) FOR FOLLOW-UP ANKYLOSING SPONDYLITIS (AS) PATIENTS WITH A RHEUMATOLOGIST-PATIENT INTERACTIVE SMART SYSTEM OF DISEASE MANAGEMENT (SSDM): A COHORT STUDY FROM CHINA}

Jing Xue ${ }^{1}$, Hui Song ${ }^{2}$, Jing Yang ${ }^{3}$, Zhenchun Zhang ${ }^{4}$, LI Hongbin ${ }^{5}$, Junli Zhang ${ }^{6}$, Zongwen Shuai ${ }^{7}$, Zhenbiao Wu ${ }^{8}$, Henglian Wu ${ }^{9}$, Bei Wang ${ }^{10}$, Hua Wei $^{11}$, LI Yasong ${ }^{12}$, LI Zhijun ${ }^{13}$, Lijun Liu ${ }^{14}$, Yuhua Jia ${ }^{15}$, Fei Xiao ${ }^{15}$, Huaxiang Wu ${ }^{1}$, SSDM Collaboration Group, China. ${ }^{1}$ The Second Affiliated Hospital of Zhejiang University School of Medicine, Hangzhou, China; ${ }^{2}$ Beijing Jishuitan Hospital, Beijing, China; ${ }^{3}$ Central Hospital of MianYang, Mianyang, China; ${ }^{4}$ Linyi people's Hospital, Linyi, China; ${ }^{5}$ The Affiliated Hospital of Inner Mongolia Medical University, Huhehaote, China; ${ }^{6}$ Xi 'an No.5 Hospital, Xi An, China; ${ }^{7}$ First Affiliated Hospital of Anhui Medical University, Hefei, China; ${ }^{8}$ The First Affiliated Hospital of The Fourth Military Medical University, Xi An, China; ${ }^{9}$ Dongguan Donghua Hospital, Dongguan, China; ${ }^{10}$ Beijing Hospital of Traditional Chinese Medicine (TCM), Beijing, China; ${ }^{11}$ Northern Jiangsu People's Hospital, Yangzhou, China; ${ }^{12}$ Zhejiang Provincal People's Hospital, Hangzhou, China; ${ }^{13}$ The First Affiliated Hospital of Bengbu Medical College, Bengbu, China; ${ }^{14}$ Tong Liao City Hospital, Tongliao, China; ${ }^{15}$ Shanghai Gothic Internet Technology Co., Ltd., Shanghai, China

Background: Ankylosing Spondylitis Disease Activity Score (ASDAS) is adopted to evaluate the degree of disease activity and the inflammatory response in AS patients. ASDAS score $\leq 1.3$ represents inactive disease status and achievement of T2T.

Objectives: To evaluate the patterns of T2T and related influential factors among AS patients after applying SSDM in the real world.

Methods: SSDM is a mobile application for disease management. Patients were trained to master SSDM by healthcare professionals and to conduc ASDAS self-assessments. Patients were also required for repeating selfevaluation after leaving the hospital. After entry by patients, data can synchronized to the SSDM terminal of authorized rheumatologists.

Results: From Jan 2015 to Jan 2019, 667 AS patients across China were followed up for more than 6 months through SSDM. The results at baseline and in final follow up were summarized in Table 1. The rate of T2T achievers were $28.49 \%(190 / 667)$ at baseline, and improved significantly to $41.38 \%(276 / 667)$ after 6 months follow up, $\mathrm{p}<0.01$. Among T2T achievers at baseline, 64.21\% (122/190) maintained T2T, 35.79\% (68/190) relapsed. Of patients who didn' t achieve T2T at baseline, only $32.29 \%$ (154/477) of the other AS patients achieved T2T after 6 months follow up. We further analyzed the impact of the times of self-assessment for ASDAS on T2T. The patients were stratified according to their frequency of self-assessment: more than 3 times, less than or equal to 3 times self-assessments within 6 months follow-up. Results show that the more frequent of the self-assessment, the higher improvement of T2T rate $(19.34 \%$ vs. $8.40 \%$, Table 2 ). A linear regression analysis of variables in statistics and parameter estimation was conducted by least square method. The improvement of T2T rate(y) was positively correlated with times of self-assessment for $\operatorname{ASDAS}(\mathrm{x})$ independently (Figure 1).

Conclusion: Significant improvement was observed under applying SSDM through empowering AS patients. After proactive disease management via SSDM for more than 6 months, Patients with ASDAS $\leq 1.3$ score at baseline had a significantly higher retention rate of disease activity. The patients who performed more self-assessments through SSDM had lower probability of relapse and higher rate of T2T. SSDM is a valuable tool for long term follow-up through empowering patients. 